\title{
Quantitative Estimation and Chemical Coding of Spiny Type I Neurons in Human Intestines
}

\author{
Julia Schuy ${ }^{a} \quad$ Anne Schlabrakowski ${ }^{b}$ Winfried Neuhuber ${ }^{a} \quad$ Axel Brehmer $^{a}$ \\ ${ }^{\mathrm{a}}$ Institute of Anatomy I and ${ }^{\mathrm{b}}$ Institute of Pathology, University of Erlangen-Nuremberg, Erlangen, Germany
}

\section{Key Words}

Enteric nervous system $\cdot$ Galanin $\cdot$ Neuronal nitric oxide synthase $\cdot$ Vasoactive intestinal peptide $\cdot$ Myenteric plexus

\begin{abstract}
Previous studies have shown that most human myenteric neurons co-staining for vasoactive intestinal peptide (VIP), neuronal nitric oxide synthase (nNOS) and neurofilaments (NF) display the morphology of spiny type I neurons displaying a descending projection pattern. Here, we estimated the proportions of spiny neurons in human intestines, the amount of congruence of VIP/nNOS-immunoreactive with spiny neurons and whether galanin (GAL) is co-localized with VIP. Three sets of colchicine-pretreated and fixed whole mounts of 21 patients or body donors (median age 65 years; 10 female, 11 male) were stained for VIP, nNOS and NF, for VIP, nNOS and the human neuronal protein $\mathrm{Hu} C / \mathrm{D}(\mathrm{HU})$ as well as for VIP, nNOS and GAL. The majority of VIP/nNOS-co-reactive neurons were spiny neurons $(79 / 80 \%$ in small/large intestine, respectively) and the majority of spiny neurons costained for VIP and nNOS (82/69\%). Neurons co-immunoreactive for VIP/nNOS/HU amounted to 7 and $4 \%$, respectively. GAL/VIP-co-immunoreactivity was demonstrated in 69 and $27 \%$ of spiny neurons, respectively. We conclude that the number of neurons displaying co-reactivity for VIP and nNOS is a quantitative indicator of spiny neurons in both small and
\end{abstract}

large intestine and that the proportion of spiny neurons is about $7 \%$ in small and $4 \%$ in large intestines. Since nerve fibres co-staining for NF/VIP/nNOS were found mainly in the circular muscle layer but not the surrounding perikarya of spiny neurons, we suggest that they may represent inhibitory motor neurons rather than descending interneurons.

Copyright $\odot 2010$ S. Karger AG, Basel

\section{Introduction}

Current routine pathology of enteric nervous tissue probes for histological diagnosis in gastrointestinal neuromuscular and other diseases mainly evaluates numbers, sizes and general cytological features of neurons and ganglia as well as their normal or ectopic localization

\section{Abbreviations used in this paper}

ChAT choline acetyltransferase

GAL galanin

$\mathrm{HU}$ human neuronal protein $\mathrm{Hu} \mathrm{C} / \mathrm{D}$

NF neurofilament

nNOS neuronal nitric oxide synthase

TBS Tris-buffered saline

VIP vasoactive intestinal peptide

\section{KARGER}

Fax +4161306 1234

E-Mail karger@karger.ch

www.karger.com
(C) 2010 S. Karger AG, Basel

$1422-6405 / 11 / 1933-0195 \$ 38.00 / 0$

Accessible online at:

www.karger.com/cto
Dr. Axel Brehmer

Institute of Anatomy I, University of Erlangen-Nuremberg

Krankenhausstrasse 9, DE-91054 Erlangen (Germany)

Tel. +49 9131852 2831, Fax +499131 8522863

E-Mail axel.brehmer@anatomiel.med.uni-erlangen.de 
Table 1. Patients, body donors and tissue characteristics

\begin{tabular}{llll}
\hline Segment & Age & Sex & Diagnosis (body donor) \\
\hline Duodenum & 42 & female & pancreas carcinoma \\
Duodenum* $^{*}$ & 67 & male & pancreas carcinoma \\
Duodenum & 84 & male & colon carcinoma \\
Jejunum* $^{*}$ & 36 & male & polytrauma \\
Jejunum* $^{*}$ & 82 & male & liver cirrhosis (body donor) \\
Jejunum & 84 & male & colon carcinoma \\
Ileum & 38 & male & diabetes mellitus (body donor) \\
Ileum & 57 & male & Meckel's diverticle \\
Ileum* & 86 & female & heart failure (body donor) \\
Colon asc.* & 52 & female & colon carcinoma \\
Colon asc. & 61 & female & colon carcinoma \\
Colon asc.* & 66 & male & colon carcinoma \\
Colon trans.* & 68 & female & colon carcinoma \\
Colon trans. & 72 & female & colon carcinoma \\
Colon trans.* & 72 & female & colon carcinoma \\
Colon desc. & 44 & female & colon carcinoma \\
Colon desc.* & 51 & male & colon carcinoma \\
Colon desc.* & 66 & female & colon carcinoma \\
Colon sig. & 27 & male & diverticulitis \\
Colon sig. & 49 & male & diverticulitis \\
Colon sig.* & 65 & female & colon carcinoma \\
\hline
\end{tabular}

asc. $=$ Ascendens; trans. $=$ transversum; desc. = descendens; sig. $=$ sigmoideum.

* From these segments, whole mounts triple-stained for VIP, GAL and NF were stained.

[Knowles et al., 2009]. One possibility to extend the spectrum of diagnostic criteria would be the evaluation of changes in proportions of different enteric neuron types. An important precondition for this refinement of diagnostic potential is the morphochemical definition of human enteric neuron types. Numerous studies in the guinea pig enteric nervous system resulted in a quite complete list of myenteric and submucosal neuron types. Their proportions, given by authors of different laboratories [Costa et al., 1996; Furness, 2006], vary only slightly.

Although the categorization of human enteric neurons started more than 100 years ago [Dogiel, 1899], our knowledge is still fragmentary [Brehmer, 2006]. One of the human myenteric neuron types described recently is co-immunoreactive for vasoactive intestinal peptide (VIP), neuronal nitric oxide synthase (nNOS) and neurofilaments (NF) and consistently displays, in contrast to other neuron types, spiny-shaped dendrites. This unique combination of chemical and morphological features led us to separate them as spiny from other Dogiel type I neurons [Brehmer et al., 2004, 2006; Lindig et al., 2009]. Most of these spiny type I neurons have anally directed axons running within the myenteric plexus as long as they can be traced. In these studies, a mainly qualitative characterization was addressed although, in a small number of samples, we estimated the proportion of VIP/nNOS-coreactive neurons in relation to neurons positive for the human neuronal protein $\mathrm{Hu} \mathrm{C} / \mathrm{D}$ (HU) [Brehmer et al., 2006]. However, it is not yet clear whether or not all spiny neurons are co-reactive for both VIP and nNOS, i.e. to what extent neurons displaying VIP/nNOS-co-reactivity match quantitatively with neurons characterized by spiny-shaped dendrites. A certain variability in the detectability of transmitters in some enteric neuron types is known from the guinea pig [Costa et al., 1996; Furness, 2006] and was also observed to a considerable extent in the human enteric nervous system [Beck et al., 2009]. It is also established that the chemical codes of neurons vary under different circumstances [De Giorgio and Camilleri, 2004; Ekblad and Bauer, 2004; Schemann and Neunlist, 2004].

It is our overall goal to distinguish human enteric neuron populations based on their morphochemical characteristics and to determine their proportions within the whole enteric (here myenteric) neuron population. The aims of the present study were 4 -fold. First, to evaluate the proportions of VIP/nNOS-co-reactive neurons in whole mounts counterstained with the panneuronal marker HU on a larger sample than previously [Brehmer et al., 2006]. Second, to estimate the amount of congruence between VIP/nNOS-co-reactive neurons and NFstained spiny neurons. Third, to determine the distribution pattern of nerve fibres co-reactive for VIP and nNOS within the gut layers. Fourth, to investigate whether or not there is a co-existence of galanin (GAL) and VIP in spiny or other myenteric neurons as it is known from laboratory animals [Stach, 1989; Furness, 2006; Jungbauer et al., 2006] and as it was also found in nerve fibres of human colonic muscle layers [Burleigh and Furness, 1990].

\section{Materials and Methods}

Tissue Processing

The use of human tissues for these experiments was approved by the Ethical Committee of the University of Erlangen-Nuremberg. In table 1 , we listed the characteristics of our tissue donors (median age 65 years; 10 females, 11 males). If patients suffered from tumours, only tissues obtained from the non-tumour-infiltrated borders of the resected gut segments were included. Segments derived from body donors could be used in those rare cases in which the post-mortem delay was maximally $6 \mathrm{~h}$. 
Table 2. Antisera

\begin{tabular}{|c|c|c|c|}
\hline Antigen & Host & Dilution & Source \\
\hline \multicolumn{4}{|l|}{ Primary antisera } \\
\hline Human neuronal protein $\mathrm{Hu} \mathrm{C} / \mathrm{D}(\mathrm{HU})$ & mouse & $1: 50$ & A21271; Mobitec (Germany) \\
\hline Neurofilament 200 (NF) & mouse & $1: 200$ & N 0142; Sigma (USA) \\
\hline Neuronal nitric oxide synthase (nNOS) & rabbit & $1: 400$ & Dr. Mayer; University of Graz (Austria) \\
\hline Vasoactive intestinal peptide (VIP) & guinea pig & $1: 500$ & 16071; Progen (Germany) \\
\hline Galanin (GAL) & rabbit & $1: 500$ & T-4326; Bachem (Germany) \\
\hline \multicolumn{4}{|l|}{ Secondary antisera } \\
\hline $\mathrm{Cy}^{\mathrm{TM}_{3} \text {, donkey anti-guinea pig } \mathrm{IgG}}$ & & $1: 1,000$ & 706-165-148; Dianova (Germany) \\
\hline ALEXA Fluor 647, donkey anti-mouse & & $1: 1,000$ & A-31571; Mobitec (Germany) \\
\hline ALEXA Fluor 647, donkey anti-rabbit & & $1: 1,000$ & A-31573; Mobitec (Germany) \\
\hline ALEXA Fluor 488, donkey anti-rabbit & & $1: 1,000$ & A-21206; Mobitec (Germany) \\
\hline ALEXA Fluor 488 , donkey anti-mouse & & $1: 1,000$ & A-21202; Mobitec (Germany) \\
\hline
\end{tabular}

Intestinal segments were transported in physiological saline ( $\mathrm{pH} 7.3$ ) on ice to the laboratory. Upon arrival (up to $6 \mathrm{~h}$ after resection), they were rinsed in Krebs solution at room temperature and transferred to Dulbecco's modified Eagle's medium (DME/ F12-Ham; Sigma Chemical Co., St. Louis, Mo., USA) containing $10 \mathrm{mg} / \mathrm{ml}$ antibiotic-antimycotic (Sigma), $50 \mu \mathrm{g} / \mathrm{ml}$ gentamycin (Sigma), $2.5 \mu \mathrm{g} / \mathrm{ml}$ amphotericin B (Sigma), 10\% foetal bovine serum (Sigma), $4 \mu \mathrm{M}$ nicardipine and $2.1 \mathrm{mg} / \mathrm{ml} \mathrm{NaHCO}$, bubbled with $95 \% \mathrm{O}_{2}$ and $5 \% \mathrm{CO}_{2}$ at $37^{\circ} \mathrm{C}$ for $1-2 \mathrm{~h}$. Thereafter, they were incubated for $2-8 \mathrm{~h}$ in the same medium with $100 \mu \mathrm{M}$ colchicine added to enhance neuropeptide immunoreactivities in the neuronal somata.

For fixation, samples were pinned out on a Sylgard-lined Petri dish and transferred to $4 \%$ formalin in $0.1 \mathrm{M}$ phosphate buffer $(\mathrm{pH}$ 7.4) at room temperature for 2-4 h. Some segments were stored at $-70^{\circ} \mathrm{C}$ in methylbutane after cryoprotection with $15 \%$ saccharose in $0.1 \mathrm{M}$ phosphate buffer.

For the following incubation steps, two longitudinal muscle/ myenteric plexus whole mounts from each segment as well as a third one from those 11 segments marked with an asterisk in table 1 were prepared.

\section{Immunohistochemistry}

Each of the 21 whole mounts was triple-stained for VIP, nNOS, HU and for VIP, nNOS, NF, whereas 11 further specimens were stained for VIP, GAL, NF (asterisks in table 1). Additionally, cryostat sections parallel to the gut longitudinal axis in undissected, frozen whole mounts were triple-stained for VIP, nNOS and NF.

Incubations of specimen included the following steps: preincubation of whole mounts for $2 \mathrm{~h}$ (sections for $1 \mathrm{~h}$ ) in $0.05 \mathrm{M}$ Trisbuffered saline (TBS; pH 7.4) containing $1 \%$ bovine serum albumin, $0.5 \%$ Triton X-100, 0.05\% thimerosal and 5\% normal donkey serum. After rinsing in TBS for $10 \mathrm{~min}$, the whole mounts were incubated in a solution containing bovine serum albumin, Triton $\mathrm{X}-100$, thimerosal (see above) and the primary antibodies (table 2) for $72 \mathrm{~h}\left(4^{\circ} \mathrm{C}\right.$; sections overnight). After an overnight rinse in TBS at $4^{\circ} \mathrm{C}$, whole mounts were incubated in an equivalent solution to that with the primary antibodies but with added secondary antibodies (table 2; $4 \mathrm{~h}$; room temperature; sections $1 \mathrm{~h}$ ) followed by a rinse in TBS (overnight; $4^{\circ} \mathrm{C}$ ).

Quantitative Estimation of Spiny

Neurons
To reduce lipofuscin-induced autofluorescence, whole mounts were incubated in ammonium acetate buffer ( $\mathrm{pH}$ 5.0) containing $1 \mathrm{mM} \mathrm{CuSO}_{4}$ for $120 \mathrm{~min}$ followed by a short rinse in distilled $\mathrm{H}_{2} \mathrm{O}$ [Schnell et al., 1999; Brehmer et al., 2004]. Thereafter, both whole mounts and sections were mounted with TBS-glycerol $(1: 1 ; \mathrm{pH}$ 8.6).

Whole mounts and sections incubated in solutions lacking primary antisera (negative controls) yielded no staining. Additionally, for the antigens VIP (Bachem, H-3775) and GAL (Peninsula, 7100), preabsorption of the two antibodies (table 2) with 2-, 10 - and 20 -fold excess of antigen was performed overnight at $4^{\circ} \mathrm{C}$. The antigen-antibody mixtures were spun at 20,000 $g$ for $20 \mathrm{~min}$ to sediment precipitating antigen-antibody complexes and avoid high background staining. The supernatant was then used in place of the primary antibody (fig. 1).

\section{Image Acquisition, Quantitative Evaluations}

Each 15 ganglia or single neurons lying outside of ganglia at interconnecting nerve strands were selected randomly from all specimens, in a meander-like fashion. Three-line z-series through myenteric ganglia ( $\mathrm{z}$-steps each $1.6 \mu \mathrm{m}$ ) were created using a confocal laser scanning microscope [Nikon Eclipse E1000-M (Tokyo, Japan) equipped with a confocal system: Nikon Digital Eclipse C1 with 3 channels, laser configuration: 488-nm argon laser, 543-nm helium-neon laser (both from Melles Griot Inc., Carlsbad, Calif., USA) and 638-nm diode laser (Coherent, Santa Clara, Calif., USA)]. A $20 \times$ dry objective lens (numerical aperture 0.75 ) was applied, and the zoom factor was set to 2.0 in all scanning sessions. All counts were carried out on these z-series, using the FreeViewer software (EZ-C1 3.30) by Nikon. We tried to carefully discriminate neurons lying at the same $x-y-b u t$ at different $\mathrm{z}$-positions to avoid false-positive recordings of neurons (e.g. one double-stained instead of two single-stained, overlapping neurons).

The figures are projections of $\mathrm{z}$-series and were prepared using Adobe Photoshop CS (8.0.1) and CorelDRAW X4. 

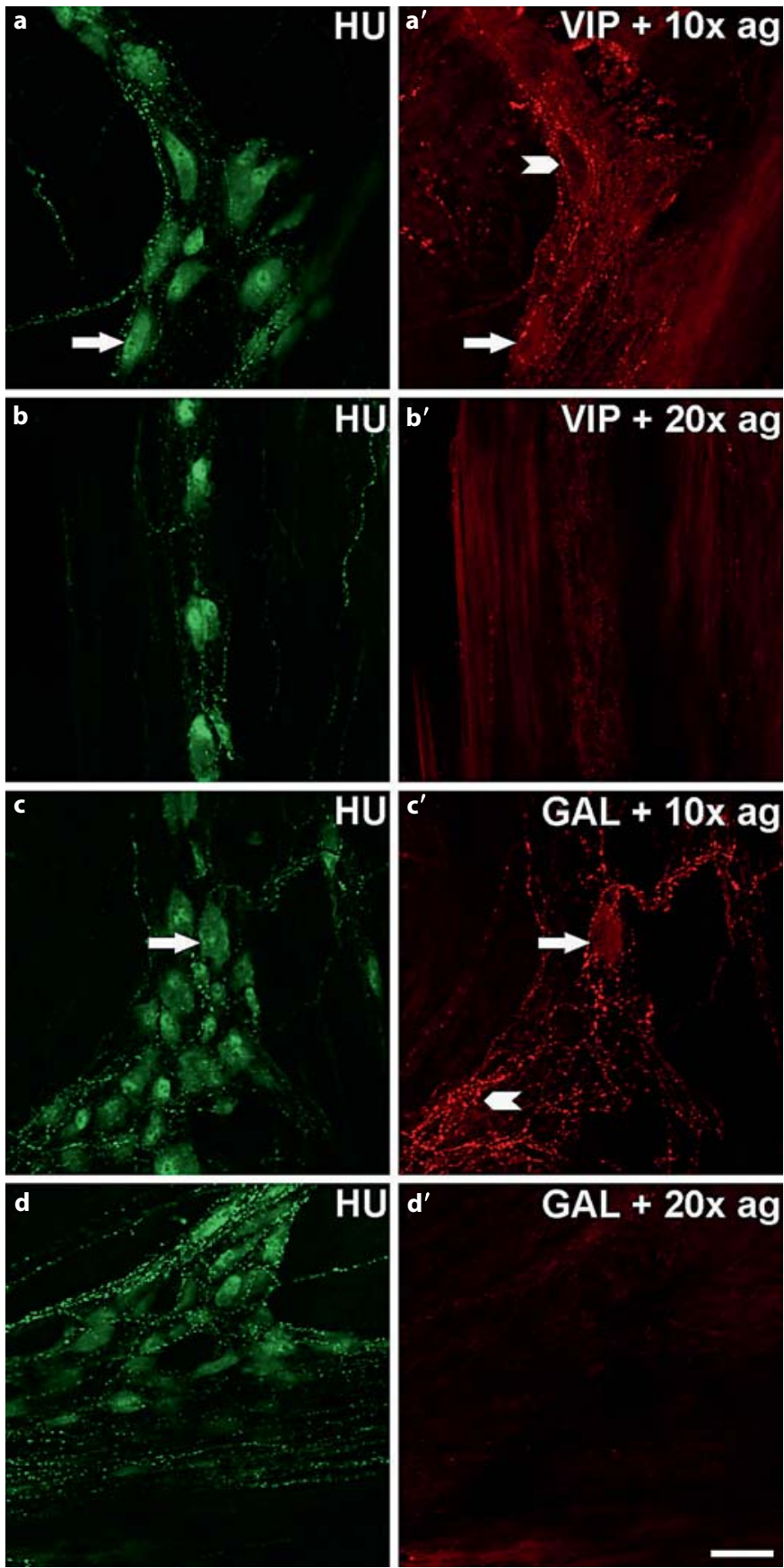

Fig. 1. Preabsorption controls for antibodies against VIP and GAL counterstained for $\mathrm{HU}$ and depicted as single optical sections through myenteric ganglia (a-d). Incubations using the supernatants after preabsorption with 10 -fold antigen (ag) excess resulted in weak neuronal labelling for both VIP and GAL. Arrows point at somata and arrowheads at nerve fibres in a' (fibres surrounding a VIP-negative nerve cell body) and $\mathbf{c}^{\prime}$, respectively. Incubation after preabsorption with 20 -fold antigen excess resulted in no specific neuronal VIP and GAL labelling, respectively (b', d'; nonneuronal background staining). Bar $=50 \mu \mathrm{m}$.

\section{Results}

Proportions of VIP- and nNOS-Reactive as well as

\section{Co-Reactive Neurons}

These were evaluated in 21 specimens triple-stained for VIP, nNOS and HU, the counts are summarized in table 3 and an example is depicted in figure $2 \mathrm{a}$.

Generally, both VIP- and nNOS-immunolabelling was cytoplasmic. This is clearly visible in single optical sections (fig. 1) but can be obscured, to some degree, in projected z-series (fig. 2-4). VIP displayed a granular staining pattern (similar to GAL), whereas nNOS appeared more homogeneous. HU intensely labelled the nuclei (without nucleoli) and, frequently but less intensely, also the cytoplasm. As observed earlier [Ganns et al., 2006], some specimens also displayed some HU-stained nerve fibres.

$\mathrm{VIP} / \mathrm{nNOS}$-co-reactive neurons amounted to $7 \%$ in the small and $4 \%$ in the large intestine. In both the small and large intestine, a major group of neurons was negative for both VIP and nNOS (61 and 53\%, respectively). Nitrergic neurons negative for VIP comprised 30 and $41 \%$. The smallest group were VIP/HU neurons with $2 \%$ in each organ. The total of nNOS-positive neurons amounted to $37 \%$ in the small and $45 \%$ in the large intestine. The total of VIP-reactive neurons comprised $9 \%$ in the small intestine and $6 \%$ in the large intestine.

We observed substantial variations, e.g. VIP/nNOS neurons occurred at a frequency between 2 and 15\% in the small intestine and between 1 and $9 \%$ in the colon. Another example was the neurons immunoreactive only for $\mathrm{HU}$ ranging between 47 and $74 \%$ in the small and between 39 and $62 \%$ in the large intestine.

Out of the 11,602 neurons recorded, 4 neurons, immunoreactive either for VIP or for nNOS, did not display reactivity for $\mathrm{HU}$ (table 3 ).

\section{Quantitative Match of VIP/nNOS-Reactive Neurons} with NF-Reactive Spiny Neurons

This was analysed in 21 specimens triple-stained for VIP, nNOS and NF. The results are summarized in table 4 , and examples are depicted in figure $2 \mathrm{~b}$ (small intestine) and figure $2 c$ (large intestine).

Spiny neurons could be morphologically identified exclusively based on their NF immunoreactivity. Regional variations were observed as described earlier [Brehmer, 2006; Brehmer et al., 2006]: in the upper small intestine, numerous spiny neurons had main dendrites, partly displaying considerable lengths. Generally, the spiny dendrites were shorter and less conspicuous in the large as compared to the small intestine. 
Table 3. Percentages of neurons immunoreactive for all 3, for 2 or for only 1 marker counted in whole mounts stained for VIP, nNOS and $\mathrm{HU}$

\begin{tabular}{|c|c|c|c|c|c|c|}
\hline Segment & $\begin{array}{l}\text { Age/ } \\
\text { gender }\end{array}$ & $\begin{array}{l}\text { VIP/ } \\
\text { nNOS/ } \\
\text { HU, \% }\end{array}$ & $\begin{array}{l}\text { nNOS/ } \\
\text { HU, \% }\end{array}$ & $\begin{array}{l}\text { VIP/ } \\
\mathrm{HU} \\
\%\end{array}$ & $\begin{array}{l}\text { HU (non- } \\
\text { VIP, non- } \\
\text { nNOS), \% }\end{array}$ & $\begin{array}{l}\text { Total } \\
\text { number } \\
(100 \%)\end{array}$ \\
\hline Duodenum & $42 / f$ & 3 & 21 & 1 & 74 & 795 \\
\hline Duodenum & $67 / \mathrm{m}$ & 2 & 33 & 1 & 64 & 489 \\
\hline Duodenum & $84 / \mathrm{m}$ & 14 & 33 & 2 & 50 & $433^{\mathrm{a}}$ \\
\hline$\Sigma$ Duodenum & & 6 & 27 & 2 & 65 & 1,717 \\
\hline Jejunum & $36 / \mathrm{m}$ & 7 & 35 & 2 & 55 & 601 \\
\hline Jejunum & $82 / \mathrm{m}$ & 9 & 18 & 1 & 72 & 542 \\
\hline Jejunum & $84 / \mathrm{m}$ & 15 & 25 & 5 & 55 & 287 \\
\hline$\Sigma$ Jejunum & & 9 & 27 & 2 & 62 & 1,430 \\
\hline Ileum & $38 / \mathrm{m}$ & 11 & 41 & 1 & 47 & 323 \\
\hline Ileum & $57 / \mathrm{m}$ & 2 & 41 & 0 & 56 & 403 \\
\hline Ileum & $86 / f$ & 10 & 29 & 2 & 59 & 255 \\
\hline$\Sigma$ Ileum & & 7 & 38 & 1 & 54 & 981 \\
\hline$\Sigma$ Small intestine & & 7 & 30 & 2 & 61 & 4,128 \\
\hline Colon asc. & $52 / f$ & 2 & 44 & 2 & 52 & 627 \\
\hline Colon asc. & $61 / f$ & 1 & 57 & 3 & 39 & 358 \\
\hline Colon asc. & $66 / \mathrm{m}$ & 6 & 42 & 1 & 52 & 562 \\
\hline$\Sigma$ Colon asc. & & 3 & 46 & 2 & 49 & 1,547 \\
\hline Colon trans. & $68 / f$ & 4 & 44 & 2 & 50 & 1,097 \\
\hline Colon trans. & $72 / f$ & 4 & 35 & 3 & 58 & $433^{b}$ \\
\hline Colon trans. & $72 / f$ & 2 & 35 & 1 & 62 & 492 \\
\hline$\Sigma$ Colon trans. & & 4 & 40 & 2 & 55 & 2,023 \\
\hline Colon desc. & $44 / \mathrm{f}$ & 3 & 41 & 1 & 55 & 640 \\
\hline Colon desc. & $51 / \mathrm{m}$ & 3 & 35 & 4 & 59 & 596 \\
\hline Colon desc. & $66 / f$ & 9 & 35 & 3 & 54 & 647 \\
\hline$\Sigma$ Colon desc. & & 5 & 37 & 2 & 56 & 1,883 \\
\hline Colon sig. & $27 / \mathrm{m}$ & 1 & 47 & 3 & 49 & 583 \\
\hline Colon sig. & $49 / \mathrm{m}$ & 2 & 41 & 1 & 55 & 694 \\
\hline Colon sig. & $65 / f$ & 6 & 43 & 1 & 50 & 745 \\
\hline$\Sigma$ Colon sig. & & 3 & 44 & 2 & 51 & 2,022 \\
\hline$\Sigma$ Large intestine & & 4 & 41 & 2 & 53 & 7,474 \\
\hline
\end{tabular}

In the right column, the total number of neurons counted in 15 ganglia are given. asc. $=$ Ascendens; trans. = transversum; desc. $=$ descendens; sig. $=$ sigmoideum; $\mathrm{m}=$ male; $\mathrm{f}=$ female.

${ }^{a}$ Includes 3 neurons negative for HU and positive for nNOS.

${ }^{\mathrm{b}}$ Includes 1 neuron negative for HU and positive for VIP.
Table 4. Counts of neurons in myenteric ganglia $(n=15)$ of whole mounts $(\mathrm{n}=21)$ stained for VIP, $\mathrm{nNOS}$ and NF

\begin{tabular}{|c|c|c|c|c|c|c|}
\hline Segment & $\begin{array}{l}\text { Age/ } \\
\text { gender }\end{array}$ & $\begin{array}{l}\text { Spiny } \\
\text { nNOS+ } \\
\text { VIP+ }\end{array}$ & $\begin{array}{l}\text { Spiny } \\
\text { nNOS+ }\end{array}$ & $\begin{array}{l}\text { Spiny } \\
\text { nNOS- } \\
\text { VIP- }\end{array}$ & $\begin{array}{l}\text { Non-spiny } \\
\text { nNOS+ } \\
\text { VIP+ }\end{array}$ & $\begin{array}{l}\text { Non- } \\
\text { spiny } \\
\text { VIP+ }\end{array}$ \\
\hline Duodenum & $42 / f$ & 24 & 5 & 2 & 2 & 4 \\
\hline Duodenum & $67 / \mathrm{m}$ & 16 & 1 & 1 & 2 & 8 \\
\hline Duodenum & $84 / \mathrm{m}$ & 19 & 0 & 0 & 16 & 9 \\
\hline$\Sigma$ Duodenum & & 59 & 6 & 3 & 20 & 21 \\
\hline Jejunum & $36 / \mathrm{m}$ & 10 & 4 & 1 & 4 & 8 \\
\hline Jejunum & $82 / \mathrm{m}$ & 28 & 2 & 0 & 14 & 6 \\
\hline Jejunum & $84 / \mathrm{m}$ & 25 & 2 & 0 & 1 & 6 \\
\hline$\Sigma$ Jejunum & & 63 & 8 & 1 & 19 & 20 \\
\hline Ileum & $38 / \mathrm{m}$ & 14 & 8 & 1 & 1 & 3 \\
\hline Ileum & $57 / \mathrm{m}$ & 13 & 5 & 1 & 2 & 0 \\
\hline Ileum & $86 / f$ & 15 & 3 & 0 & 2 & 6 \\
\hline$\Sigma$ Ileum & & 42 & 16 & 2 & 5 & 9 \\
\hline$\Sigma$ Small intestine & & 164 & 30 & 6 & 44 & 50 \\
\hline Colon asc. & $52 / f$ & 32 & 5 & 0 & 5 & 14 \\
\hline Colon asc. & $61 / f$ & 4 & 16 & 1 & 0 & 9 \\
\hline Colon asc. & $66 / \mathrm{m}$ & 26 & 1 & 3 & 5 & 12 \\
\hline$\Sigma$ Colon asc. & & 62 & 22 & 4 & 10 & 35 \\
\hline Colon trans. & $68 / f$ & 9 & 6 & 0 & 5 & 13 \\
\hline Colon trans. & $72 / f$ & 13 & 7 & 2 & 1 & 5 \\
\hline Colon trans. & $72 / f$ & 35 & 1 & 0 & 4 & 6 \\
\hline$\Sigma$ Colon trans. & & 57 & 14 & 2 & 10 & 24 \\
\hline Colon desc. & $44 / \mathrm{f}$ & 10 & 1 & 1 & 4 & 2 \\
\hline Colon desc. & $51 / \mathrm{m}$ & 27 & 4 & 2 & 6 & 10 \\
\hline Colon desc. & $66 / f$ & 7 & 3 & 0 & 2 & 5 \\
\hline$\Sigma$ Colon desc. & & 44 & 8 & 3 & 12 & 17 \\
\hline Colon sig. & $27 / \mathrm{m}$ & 2 & 16 & 1 & 0 & 10 \\
\hline Colon sig. & $49 / \mathrm{m}$ & 14 & 4 & 1 & 4 & 9 \\
\hline Colon sig. & $65 / f$ & 5 & 1 & 1 & 9 & 15 \\
\hline$\Sigma$ Colon sig. & & 21 & 21 & 3 & 13 & 34 \\
\hline$\Sigma$ Large intestine & & 184 & 65 & 12 & 45 & 110 \\
\hline
\end{tabular}

asc. = Ascendens; trans. = transversum; desc. = descendens; sig. = sigmoideum; $\mathrm{m}=$ male; $\mathrm{f}=$ female .

We evaluated spiny neurons and their co-immunoreactivity for both VIP and nNOS (second column), only for nNOS (third column) or for none of them (fourth column). Additionally, nonspiny neurons immunoreactive for both VIP and nNOS (fifth column) and for VIP only (sixth column) were counted. 

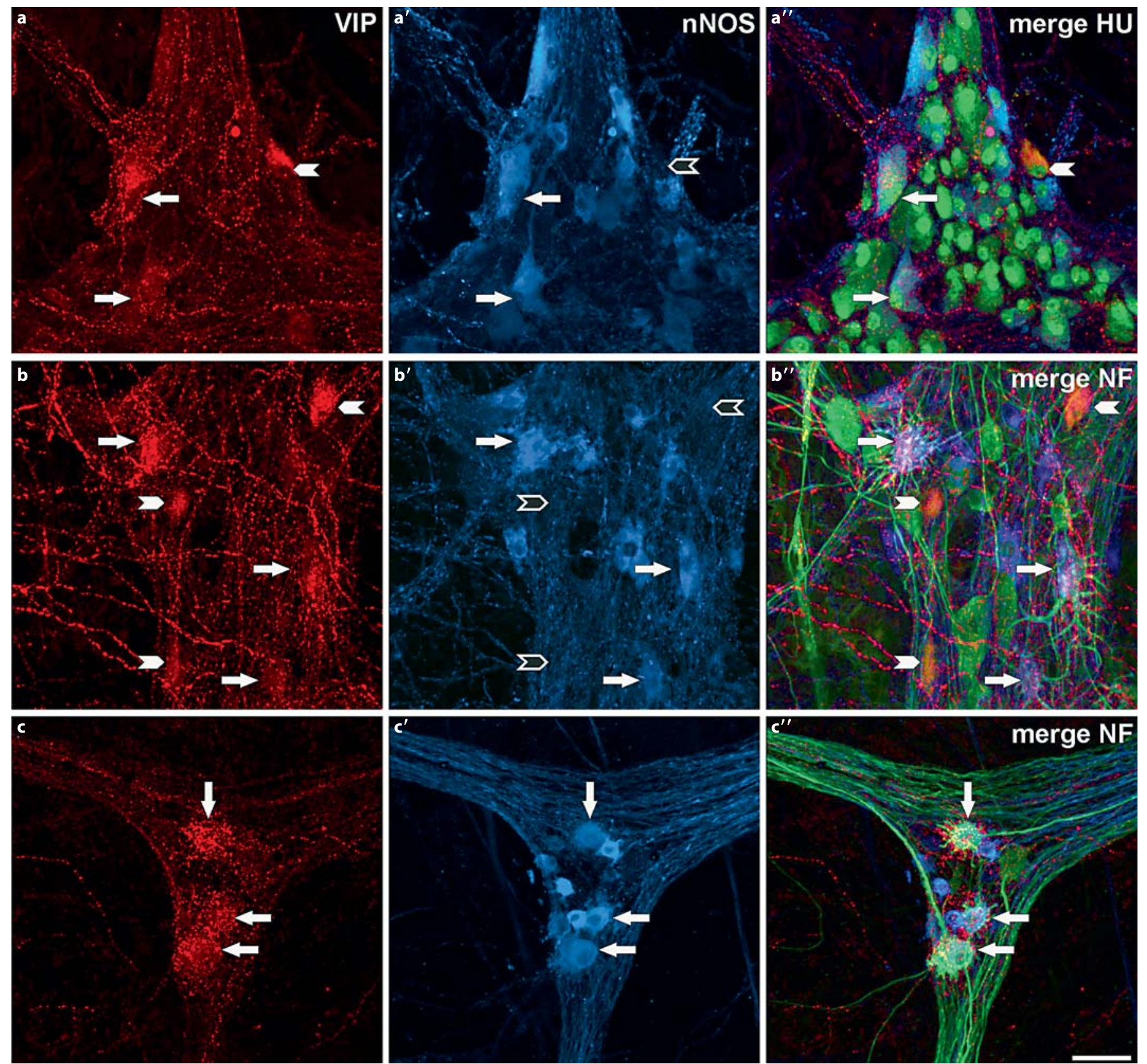

Fig. 2. Co-localization of VIP and nNOS with HU (a) or NF (b, c) depicted as projected z-series. a Two neurons are co-immunoreactive for all 3 markers (arrows point at the nuclear regions, respectively, which are stained only by $\mathrm{HU}$ ). One further neuron is positive for both VIP and HU (filled arrowheads) but negative for nNOS (empty arrowhead). Some neurons are co-reactive for nNOS and HU (blue neurons in $\mathbf{b}^{\prime}$ ) but not for VIP (not marked;

specimen from colon descendens, 66-year-old female). b Three spiny neurons co-reactive for VIP, nNOS and NF (arrows). Three further neurons are co-reactive for both VIP and NF (filled arrowheads in $\mathbf{b}$ and $\mathbf{b}^{\prime \prime}$ ) but negative for nNOS (empty arrowheads in b'; jejunum, 82-year-old male). c Three spiny neurons co-reactive for all 3 markers (arrows; colon ascendens, 66-year-old male). Bar $=50 \mu \mathrm{m}$. 
Table 5. Counts of neurons in myenteric ganglia $(n=15)$ of whole mounts $(\mathrm{n}=11)$ stained for VIP, GAL and NF

\begin{tabular}{|c|c|c|c|c|c|c|c|}
\hline Segment & $\begin{array}{l}\text { Age/ } \\
\text { gender }\end{array}$ & $\begin{array}{l}\text { Spiny } \\
\text { VIP+ } \\
\text { GAL+ }\end{array}$ & $\begin{array}{l}\text { Spiny } \\
\text { VIP+ } \\
\text { GAL- }\end{array}$ & $\begin{array}{l}\text { Spiny } \\
\text { VIP- } \\
\text { GAL- }\end{array}$ & $\begin{array}{l}\text { Non- } \\
\text { spiny } \\
\text { VIP+ } \\
\text { GAL+ }\end{array}$ & $\begin{array}{l}\text { Non- } \\
\text { spiny } \\
\text { VIP+ } \\
\text { GAL- }\end{array}$ & $\begin{array}{l}\text { Non- } \\
\text { spiny } \\
\text { VIP- } \\
\text { GAL+ }\end{array}$ \\
\hline Duodenum & $67 / \mathrm{m}$ & 15 & 6 & 5 & 2 & 3 & 0 \\
\hline Jejunum & $36 / \mathrm{m}$ & 13 & 7 & 10 & 1 & 8 & 1 \\
\hline Jejunum & $82 / \mathrm{m}$ & 34 & 1 & 0 & 12 & 8 & 9 \\
\hline Ileum & $86 / f$ & 10 & 2 & 0 & 4 & 4 & 0 \\
\hline \multicolumn{2}{|c|}{$\Sigma$ Small intestine } & 72 & 16 & 15 & 19 & 23 & 10 \\
\hline Colon asc. & $52 / \mathrm{f}$ & 0 & 3 & 8 & 4 & 4 & 1 \\
\hline Colon asc. & $66 / \mathrm{m}$ & 4 & 11 & 3 & 2 & 6 & 2 \\
\hline Colon trans. & $68 / f$ & 7 & 8 & 4 & 4 & 7 & 6 \\
\hline Colon trans. & $72 / f$ & 6 & 7 & 4 & 10 & 4 & 0 \\
\hline Colon desc. & $51 / \mathrm{m}$ & 1 & 5 & 0 & 7 & 17 & 3 \\
\hline Colon desc. & $66 / f$ & 7 & 3 & 7 & 11 & 0 & 1 \\
\hline Colon sig. & $65 / f$ & 1 & 2 & 5 & 2 & 0 & 1 \\
\hline \multicolumn{2}{|c|}{$\Sigma$ Large intestine } & 26 & 39 & 31 & 40 & 38 & 14 \\
\hline
\end{tabular}

asc. $=$ Ascendens; trans. $=$ transversum; desc. = descendens; sig. = sigmoideum; $\mathrm{m}=$ male; $\mathrm{f}=$ female.

We evaluated spiny neurons and their co-immunoreactivity for both VIP and GAL (second column), only for VIP (third column) or for none of them (fourth column). Additionally, nonspiny neurons immunoreactive for both VIP and GAL (fifth column), for VIP only (sixth column) and for GAL only (seventh column) were counted.

Taking together all spiny neurons of the $15 \times 21$ ganglia observed, we identified 200 and 265 in the small and large intestines, respectively. In the small intestinal samples, we did not find a single spiny neuron positive for VIP but not for nNOS. In contrast, in the colonic specimens, we observed 4 spiny neurons immunoreactive for VIP but not for nNOS (included in the right column of table 4). In both intestinal organs, most spiny neurons were immunoreactive for both VIP and nNOS $(82 \%$ in the small, $69 \%$ in the large intestine). Spiny neurons reactive only for nNOS accounted for $15 \%$ in the small and $25 \%$ in the large intestine. Few spiny neurons were nonreactive for both VIP and nNOS (3 vs. 5\%).

Summarizing all neurons co-immunoreactive for both VIP and nNOS, we counted 208 in the small and 229 in the large intestine. Of these, 79 and $80 \%$ were spiny neurons in the small and large intestine, respectively. VIP/ nNOS co-reactive, non-spiny neurons mostly displayed no or weak NF reactivity. In other words, we were not able to identify a common, structural feature of these neurons.
Similarly, most neurons reactive only for VIP displayed no NF reactivity. As mentioned above, 4 neurons were NF-reactive and displayed spiny-shaped dendrites. Furthermore, some VIP neurons (19 in small, 7 in large intestine; included in the right column of table 4) were moderately NF reactive and displayed very thin, gracile, hairy-like dendrites differing in shape from those of spiny neurons.

In these specimens we desisted from counting neurons immunoreactive for nNOS but negative for VIP. These were generally not or weakly NF-reactive (blue neurons in fig. $2 b^{\prime \prime}, c^{\prime \prime}$.

\section{Spiny Neurons: Co-Immunoreactivity for GAL}

We analysed 11 specimens (table 5) triple-stained for VIP, GAL and NF. An example from the small intestine is depicted in figure 3.

In the 4 small intestinal samples, we counted 91 neurons co-immunoreactive for VIP and GAL. The majority $(72=79 \%)$ were spiny neurons, the remaining $19(21 \%)$ were not or weakly stained for NF. Altogether, we found 104 spiny neurons in the small intestinal samples. Of these, 72 (69\%) displayed co-reactivity for VIP and GAL, $16(15 \%)$ were reactive only for VIP, 15 (14\%) displayed neither VIP nor GAL reactivity and 1 neuron (1\%; not included in table 5) was positive only for GAL.

In the 7 large intestinal specimens, 66 neurons were co-reactive for VIP and GAL. Only a minority of them $(26=39 \%)$ were spiny neurons, the remaining 40 neurons (61\%) were either NF-negative or were NF-positive neurons displaying heterogeneous structural features. Altogether 96 spiny neurons were found in these specimens; of these 26 (27\%) were co-reactive for VIP and GAL, 39 (41\%) displayed VIP but not GAL reactivity and 31 (32\%) showed no reactivity for either peptide.

Distribution Pattern of VIP/nNOS-Co-Reactive Nerve Fibres in Whole Mounts and Cross Sections

Most spiny neurons evaluated in our whole-mount specimens were not surrounded by VIP/nNOS varicosities (fig. $4 \mathrm{a}-\mathrm{c}$ ). Only occasionally were stained nerve fibres close to perikarya or dendrites of spiny neurons (e.g. the uppermost neuron indicated by an arrow in fig. $\left.2 b^{\prime \prime}\right)$.

In cross sections, we found abundant nerve fibres costained for VIP/nNOS and NF in the circular and, less frequently, in the longitudinal muscle layer (fig. 4d). 

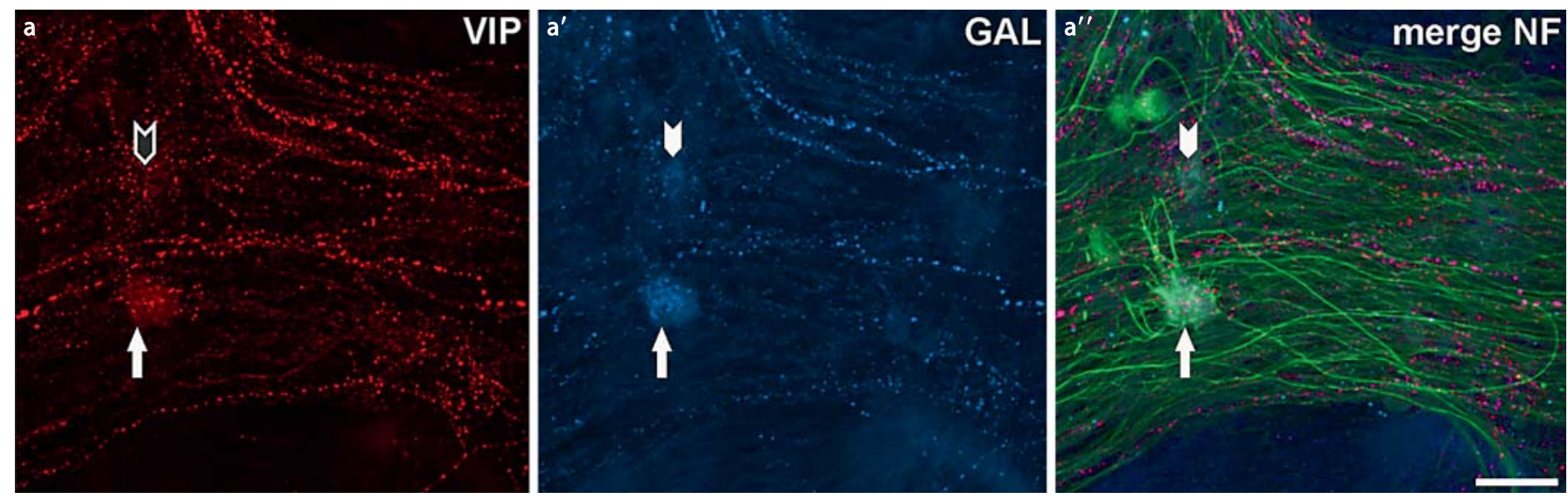

Fig. 3. Co-localization of VIP (a), GAL ( $\left.\mathbf{a}^{\prime}\right)$ and NF (a') in a spiny neuron (arrows). Another neuron is immunopositive for GAL, weakly reactive for NF but negative for VIP (arrowheads) (projected z-series; duodenum, 67-year-old male). Bar $=50 \mu \mathrm{m}$.

\section{Discussion}

In this study, we found that about four fifths of all spiny neurons, identified by their dendritic architecture revealed by strong NF reactivity, display co-reactivity for VIP and nNOS. Vice versa, the majority of VIP/nNOSco-reactive neurons are morphologically spiny neurons. As to GAL in spiny neurons, we found a remarkable difference between small and large intestines. In the small intestine, a majority of spiny neurons co-stained for GAL (69\%), whereas in the large intestine only $27 \%$ did so. Nerve fibres co-reactive for VIP/nNOS/NF were abundant in the circular muscle layer, less frequent in the longitudinal layer and were mostly absent around cell bodies and dendrites of spiny neurons.

With respect to one of the two (qualitative) main aspects of our study, i.e. immunohistochemistry, we took advantage of colchicine pretreatment prior to fixation. For decades this method has frequently been used in neuroscience [Mundy and Tilson, 1990] to enhance neuropeptide concentration (here VIP and GAL) and, thus, their immunohistochemical detectabilities within neuronal somata by blocking axonal transport. We found no effect of colchicine preincubation on the numbers of either cholinergic or nitrergic neurons [Beck et al., 2009]. As to the other aspect of our study, i.e. neuronal morphology, we reported earlier that we did not observe marked morphological changes of myenteric neurons in colchicine-treated versus non-treated whole mounts [Brehmer et al., 2004].

\section{Chemical Coding of Spiny Neurons: NF/nNOS/}

\section{$V I P / \pm G A L / \pm$ Choline Acetyltransferase}

In our previous study [Brehmer et al., 2006] we reported that neurons co-immunoreactive for VIP/nNOS/NF display common morphological features, i.e. they have one single axon mostly running anally within the myenteric plexus and display spiny-shaped dendrites. Here, we addressed the problem of congruence between VIP/ nNOS-co-reactive neurons on the one hand and spiny neurons on the other. Our study revealed two remarkable minor populations. First, neurons immunoreactive for VIP and nNOS but with no or weak NF reactivity (about one fifth of VIP/nNOS-co-reactive neurons). With our

Fig. 4. Distribution pattern of nerve fibres co-immunoreactive for VIP, nNOS and NF. a-a", $\mathbf{b}-\mathbf{b}^{\prime \prime}$ and $\mathbf{c}-\mathbf{c}^{\prime \prime}$ display projected $\mathrm{z}$-series

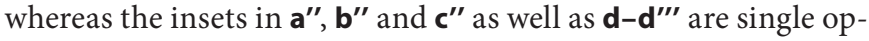
tical sections. a-c Three spiny neurons (arrows point at their axons) are represented. There is no striking neighbourhood of nerve fibres and boutons co-immunoreactive for VIP and nNOS surrounding the nerve cell bodies and dendrites of the spiny neurons [jejunum, 82-year-old male (a); duodenum, 42-year-old female (b, c)]. d Cross section through the muscular coat revealed numerous nerve fibres in the circular (two are indicated by arrowheads) and less numerous in the longitudinal (arrow) muscle layers co-immunoreactive for VIP, nNOS and NF. The area of the inset in $\mathbf{d}^{\prime \prime}$ is enlarged in d'"; arrowheads point at nerve fibres co-localizing all three markers (false colour $=$ white; duodenum, 42-year-old female). Bars $=50 \mu \mathrm{m}$ (insets $=25 \mu \mathrm{m})(\mathbf{a}-\mathbf{c}), 100 \mu \mathrm{m}\left(\mathbf{d}-\mathbf{d}^{\prime \prime}\right), 50$ $\mu \mathrm{m}\left(\mathbf{d}^{\prime \prime \prime}\right)$. 


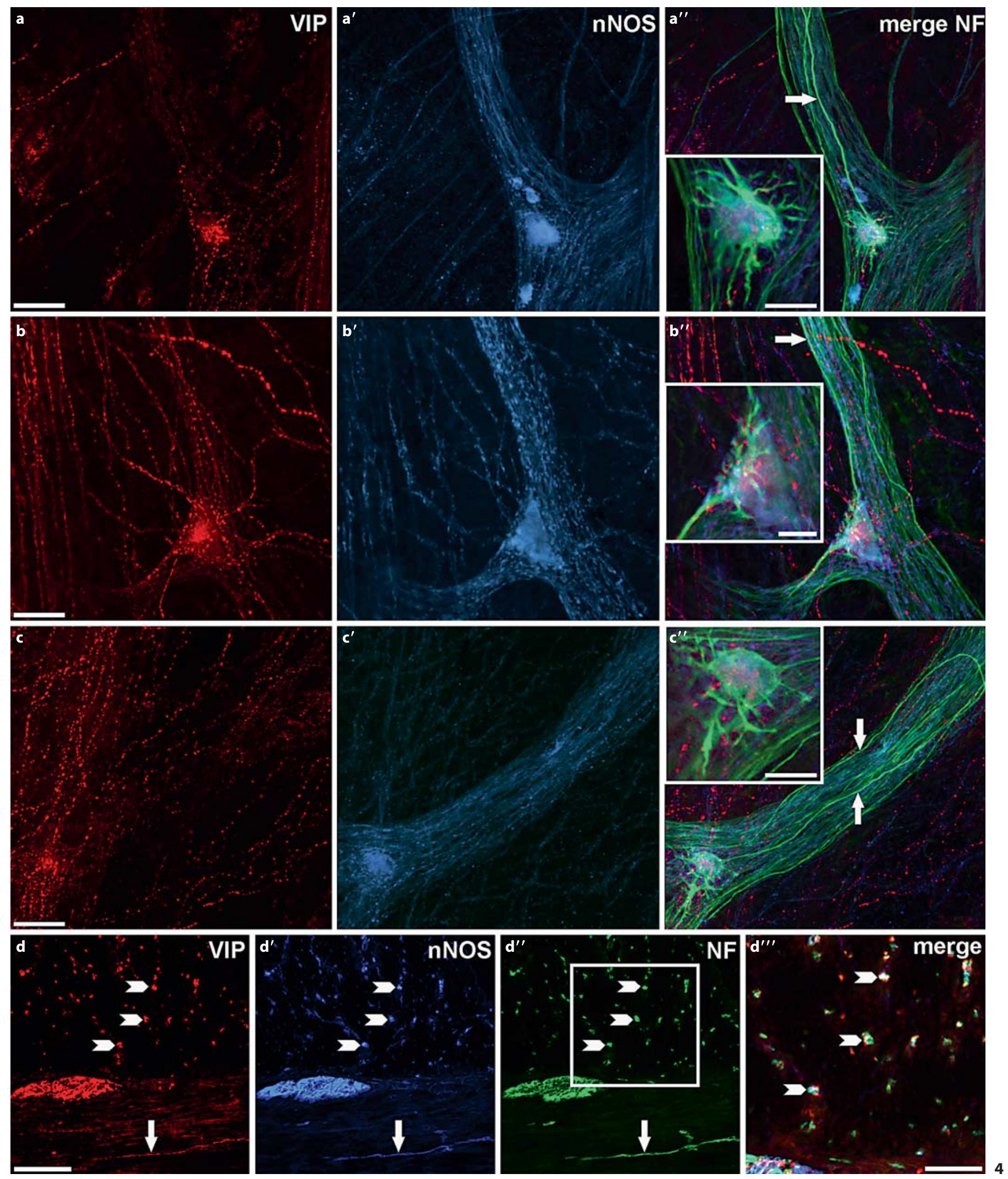


methods applied so far, we cannot answer the question whether these are different from the spiny neurons or, probably for methodological reasons, are spiny neurons which were incompletely stained for NF. Recently [Beck et al., 2009], we have argued that using human (gastrointestinal) tissues has to deal with much more heterogeneous features than using those of laboratory animals. One of the difficulties is the quite different amount of connective tissue and thickness of whole mounts derived from various individuals. This results not only in different staining intensities between tissues from different individuals but also within a given whole mount at various locations. The NF antibody seems to be one of the more slowly penetrating antibodies. Future studies will investigate the applicability of alternative structural markers for the immunohistochemical representation of spiny and other myenteric neuron types.

Besides VIP, nNOS and NF, this study showed also GAL to be present in spiny neurons. However, we found this peptide in most spiny neurons only in the small intestine, whereas in the colonic segments it occurred only in about one quarter of these neurons. Furthermore, Beck et al. [2009] showed that choline acetyltransferase (ChAT), the enzyme-generating acetylcholine, is present in about one third of spiny neurons. Thus, in the small intestine, most spiny neurons contain NF, nNOS, VIP and GAL and a minority also ChAT. In contrast, in the colon, NF, nNOS and VIP are present in a majority of spiny neurons, whereas GAL and ChAT could be found only in a minority.

\section{Proportion of Spiny Neurons: 7 and 4\%}

In the small intestinal samples of this study, about four fifths of VIP/nNOS neurons were spiny neurons (79\%). On the other hand, about four fifths of spiny neurons were co-immunoreactive for VIP and nNOS (82\%). That is, in the small intestine, the proportion of VIP/ nNOS neurons (7\%) can be used as an indicator for the proportion of spiny neurons. In a previous study [Brehmer et al., 2006], based on a much smaller sample, the proportion of VIP/nNOS neurons was similar (8.7\%). Thus, the results of both studies are in mutual accordance and the proportion of spiny neurons in the small intestine appears to be $7 \%$. This proportion is somewhat lower than that of the multiaxonal Dogiel type II, putative primary afferent neurons estimated at $10 \%$ based on comparable morphochemical characteristics [Weidmann et al., 2007].

In the large intestine, the situation is less clear for two reasons. First, whereas $80 \%$ of VIP/nNOS are spiny neu- rons, only $69 \%$ of spiny neurons contained both VIP and nNOS in the present study. Second, the proportion of $\mathrm{VIP} / \mathrm{nNOS}$ neurons determined in the previous study [Brehmer et al., 2006] differs considerably from the present value (13 vs. $4 \%$ ). Since we used a larger number of samples here, it may be that our present value is more representative. The proportion of spiny neurons in the colon is rather somewhat lower than in the small intestine, about $4 \%$, based on the present results. With regard to this quantitative difference between results of our previous [Brehmer et al., 2006] and the present study, we highlight the need for quantitative studies based on a large sample. To characterize divergent innervation patterns of the human enteric nervous system under pathological circumstances, reliable mean values obtained from a high number of individuals are necessary.

However, chemical codes of neurons vary under experimental or pathological circumstances [De Giorgio and Camilleri, 2004; Schemann and Neunlist, 2004] including even nNOS and VIP [Szabó, 1996; Ekblad and Bauer, 2004]. Thus, the varying values between individuals may reflect different states of disease and/or habits in their daily life. Future studies should address the question which types of neurons are able to express additional neuroactive substances, such as VIP, under specific circumstances.

\section{Function of Spiny Neurons: Inhibitory Motor rather than Descending Interneurons}

In the guinea pig small intestine, about $18 \%$ of myenteric neurons represent inhibitory motor neurons to both the circular and longitudinal muscle layer and contain both VIP and nNOS [Furness, 2006]. Both substances interact in the transmission of inhibitory signals from nerve via interstitial cells of Cajal onto smooth muscle [Lecci et al., 2002; Van Geldre and Lefebvre, 2004]. Although the existence of interspecies differences even in the chemical coding of enteric neurons is beyond doubt [Gershon, 1994], this does not seem to apply to the general context of smooth muscle relaxation with VIP and nNOS [Olsson and Holmgren, 2001] that has also been shown in humans [Grider, 1989; Maggi et al., 1990; Stark et al., 1993]. GAL has also effects on intestinal muscle contractility [Pothoulakis, 2000] and different experimental approaches revealed either inhibitory [Bauer et al., 1989] or excitatory effects [Burleigh and Furness, 1990]. Furthermore, one of the three GAL receptors known so far was found on human colonic epithelial cells and binding of GAL to these receptors stimulated chloride secretion [Benya et al., 1998]. 
A smaller myenteric, VIP/nNOS-co-reactive subpopulation in the guinea pig is descending interneurons amounting to about 5\% [Furness, 2006]. These (and other) interneurons form chains of like neurons in the guinea pig myenteric plexus [Young et al., 1995]. Combined tracing and immunohistochemical studies in human colon revealed that neurons containing VIP and/or nNOS project both to the circular muscle [Porter et al., 1997] and within the myenteric plexus [Wattchow et al., 1997]. In our material, nerve fibres displaying the chemical code of spiny neurons (i.e. NF/nNOS/VIP) were abundant in the musculature but sparsely found around cell bodies of spiny neurons. This indicates that most spiny neurons do not receive synaptic inputs from nNOS/VIP-co-reactive nerve terminals, i.e. from other spiny neurons. We suggest that the axons of spiny neurons, which can be observed to run anally within the myenteric plexus as long as they can be traced, enter the (circular and longitudinal?) muscle plexus after a longer anal run. Thus, spiny neurons rather represent descending inhibitory motor than descending interneurons in the above sense, i.e. they do not form descending chains of like neurons. However, we cannot exclude that they may synapse on non-spiny neurons.

Except for the 4 neurons displaying spiny dendrites and VIP but not nNOS reactivity in colonic samples, spiny neurons in our material generally displayed nNOS reactivity and the majority of them additional VIP reactivity. In other words, 15 and $25 \%$ of spiny neurons in small and large intestines, respectively, contained nNOS but not VIP. Future studies should attempt to identify neurons that develop an additional VIPergic phenotype under pathological conditions [De Giorgio and Camilleri, 2004; Ekblad and Bauer, 2004; Schemann and Neunlist, 2004]: non-VIPergic spiny or nitrergic, NF-negative non-spiny or other neurons.

\section{Usefulness of Results in Histopathological Diagnosis?}

As stated above, our overall aim is to distinguish human enteric neuron populations based on morphochemical characteristics and to estimate their proportions. For this purpose, whole-mount preparations are superior over sections. Whole mounts make it possible to evaluate morphological features of enteric neurons and to study a comparatively large number of neurons. In contrast, histopathological diagnosis of intestinal tissues is mainly based on routine sections through the gut wall or on small biopsy samples. One main result of our study is that co-existence of VIP, nNOS and NF is very likely confined to one particular neuron population, i.e. spiny neurons which probably act as inhibitory motor neurons. Another example is the co-existence of calretinin and somatostatin which points at putative primary afferent neurons in human small intestine [Weidmann et al., 2007]. These particular co-localizations of markers can also be demonstrated on sections and may allow, in the future, to detect changes in proportions of enteric neurons under pathological conditions.

\section{Summary and Conclusion}

In our earlier study [Brehmer et al., 2006], VIP/nNOSco-reactive neurons amounted to about 6-11\% (small intestine) and about 10-17\% (large intestine) of the whole myenteric neuron population. In the present study, on a much larger sample, we estimated 7 and $4 \%$ (in small and large intestines, respectively). Thus, in the small intestine, we confirmed our earlier results, whereas in the large intestine, we corrected them. In the earlier study, we found that almost all human, myenteric neurons co-reactive for nNOS, VIP and NF display the morphology of spiny neurons. On the other hand, the present study showed that the majority of morphological spiny neurons is co-reactive for nNOS and VIP (82 and 69\% in small and large intestines, respectively) and that there is a substantial minority of spiny neurons (15 vs. $25 \%$ ), displaying nNOS but not VIP reactivity. Additionally, the present study demonstrated GAL in spiny neurons (in 69\% of small intestinal and in $27 \%$ of colonic spiny neurons). In the earlier study, we observed the general, anally directed course of the axons of spiny neurons. The present study demonstrated the distribution pattern of nNOS/VIP/NFco-reactive axons within the gut wall. This pattern indicates that spiny neurons may represent inhibitory motor rather than descending interneurons.

\section{Acknowledgements}

The excellent technical assistance of Karin Löschner, Stephanie Link, Andrea Hilpert, Hedwig Symowski and Inge Zimmermann is gratefully acknowledged. Furthermore, we would like to thank Anne Stab, Tony Simpson, Jens Walluschek and Philip Eichhorn (all Erlangen) as well as Holger Rupprecht (Fürth) for their kind cooperation. We also thank Patty and Tony Simpson for linguistic advice. The study was supported by the Deutsche Forschungsgemeinschaft (BR 1815/4-1). 


\section{References}

Bauer, F.E., A. Zintel, M.J. Kenny, D. Galder, M.A. Ghatel, S.R. Bloom (1989) Inhibitory effect of galanin on postprandial gastrointestinal motility and gut hormone release in humans. Gastroenterology 97: 260-264.

Beck, M., A. Schlabrakowski, F. Schrödl, W. Neuhuber, A. Brehmer (2009) ChAT and NOS in human myenteric neurons: co-existence and co-absence. Cell Tissue Res 338: 37-51.

Benya, R.V., K.A. Matkowskyi, A. Danilkovich, G. Hecht (1998) Galanin causes $\mathrm{Cl}^{-}$secretion in the human colon. Potential significance of inflammation-associated NF-kappa $B$ activation on galanin-1 receptor expression and function. Ann NY Acad Sci 863: 64-77.

Brehmer, A. (2006) Structure of enteric neurons. Adv Anat Embryol Cell Biol 186: 1-94.

Brehmer, A., B. Blaser, G. Seitz, F. Schrödl, W Neuhuber (2004) Pattern of lipofuscin pigmentation in nitrergic and non-nitrergic neurofilament immunoreactive myenteric neuron types of human small intestine. Histochem Cell Biol 121: 13-20.

Brehmer, A., F. Schrödl, W. Neuhuber (2006) Morphology of VIP/nNOS-immunoreactive myenteric neurons in the human gut. Histochem Cell Biol 125: 557-565.

Burleigh, D.E., J.B. Furness (1990) Distribution and actions of galanin and vasoactive intestinal peptide in the human colon. Neuropeptides 16: 77-82.

Costa, M., S.J. Brookes, P.A. Steele, I. Gibbins, E. Burcher, C.J. Kandiah (1996) Neurochemical classification of myenteric neurons in the guinea-pig ileum. Neuroscience 75: 949-967.

De Giorgio, R., M. Camilleri (2004) Human enteric neuropathies: morphology and molecular pathology. Neurogastroenterol Motil 16: 515-531

Dogiel, A.S. (1899) Ueber den Bau der Ganglien in den Geflechten des Darmes und der Gallenblase des Menschen und der Säugethiere. Arch Anat Physiol Anat Abt (Leipzig) 130 158.

Ekblad, E., A.J. Bauer (2004) Role of vasoactive intestinal peptide and inflammatory mediators in enteric neuronal plasticity. Neurogastroenterol Motil 16(suppl 1): 123-128.
Furness, J.B. (2006) The Enteric Nervous System. Oxford, Blackwell.

Ganns, D., F. Schrödl, W. Neuhuber, A. Brehmer (2006) Investigation of general and cytoskeletal markers to estimate numbers and proportions of neurons in the human intestine. Histol Histopathol 21: 41-51.

Gershon, M.D., A.L. Kirchgessner, P.R. Wade (1994) Functional anatomy of the enteric nervous system; in Johnson, L.R. (ed): Physiology of the Gastrointestinal Tract. New York, Raven Press, pp 381-422.

Grider, J.R. (1989) Identification of neurotrans mitters regulating intestinal peristaltic reflex in humans. Gastroenterology 97: 14141419.

Jungbauer, C., T.M. Lindig, F. Schrödl, W. Neuhuber, A. Brehmer (2006) Chemical coding of myenteric neurons with different axonal projection patterns in the porcine ileum. J Anat 209: 733-743.

Knowles, C.H., R. De Giorgio, R.P. Kapur, E. Bruder, G. Farrugia, K. Geboes, M.D. Gershon, J. Hutson, G. Lindberg, J.E. Martin, W.A. Meier-Ruge, P.J. Milla, V.V. Smith, J.M. Vandervinden, B. Veress, T. Wedel (2009) Gastrointestinal neuromuscular pathology: guidelines for histological techniques and reporting on behalf of the Gastro 2009 International Working Group. Acta Neuropathol 118: 271-301

Lecci, A., P. Santicioli, C.A. Maggi (2002) Pharmacology of transmission to gastrointestinal muscle. Curr Opin Pharmacol 2: 630-641.

Lindig, T.M., V. Kumar, R. Kikinis, S. Pieper, F. Schrödl, W.L. Neuhuber, A. Brehmer (2009) Spiny versus stubby: $3 \mathrm{D}$ reconstruction of human myenteric (type I) neurons. Histochem Cell Biol 131: 1-12.

Maggi, C.A., R. Patacchini, P. Santicioli, S. Giuliani, D. Turini, G. Barbanti, A. Giachetti, A. Meli (1990) Human isolated ileum: motor responses of the circular muscle to electrical field stimulation and exogenous neuropeptides. Naunyn Schmiedebergs Arch Pharmacol 341: 256-261.

Mundy, W.R., H.A. Tilson (1990) Neurotoxic effects of colchicine. Neurotoxicology 11: 539547.
Olsson, C., S. Holmgren (2001) The control of gut motility. Comp Biochem Physiol A Mol Integr Physiol 128: 481-503.

Porter, A.J., D.A. Wattchow, S.J. Brookes, M Costa (1997) The neurochemical coding and projections of circular muscle motor neurons in the human colon. Gastroenterology 113: 1916-1923.

Pothoulakis, C. (2000) The role of neuroenteric hormones in intestinal infectious diseases. Curr Opin Gastroenterol 16: 536-540.

Schemann, M., M. Neunlist (2004) The human enteric nervous system. Neurogastroenterol Motil 16(suppl 1): 55-59.

Schnell, S.A., W.A. Staines, M.W. Wessendorf (1999) Reduction of lipofuscin-like autofluorescence in fluorescently labeled tissue. J Histochem Cytochem 47: 719-730.

Stach, W. (1989) A revised morphological classification of neurons in the enteric nervous system; in Singer, M.V., H. Goebell (eds) Nerves and the Gastrointestinal Tract. Lancaster, Kluwer, pp 29-45.

Stark, M.E., A.J. Bauer, M.G. Sarr, J.H. Szurszewski (1993) Nitric oxide mediates inhibitory nerve input in human and canine jejunum Gastroenterology 104: 398-409.

Szabó, C. (1996) Physiological and pathophysiological roles of nitric oxide in the central nervous system. Brain Research Bulletin 41: 131-141.

Van Geldre, L.A., R.A. Lefebvre (2004) Interaction of NO and VIP in gastrointestinal smooth muscle relaxation. Curr Pharm Des 10: 2483-2497.

Wattchow, D.A., A.J. Porter, S.J. Brookes, M Costa (1997) The polarity of neurochemically defined myenteric neurons in the human colon. Gastroenterology 113: 497-506.

Weidmann, S., F. Schrödl, W. Neuhuber, A. Brehmer (2007) Quantitative estimation of putative primary afferent neurons in the myenteric plexus of human small intestine. Histochem Cell Biol 128: 399-407.

Young, H.M., J.B. Furness, J.M. Povey (1995) Analysis of connections between nitric oxide synthase neurons in the myenteric plexus of the guinea-pig small intestine. J Neurocytol 24: 257-263. 